\section{Eine kohärente ärztliche Weiter- und Fortbildung für Management und Qualitätsentwicklung}

\author{
P. Berchtold
}

vielerorts herrscht Unsicherheit darüber, welche zusätzlichen Kenntnisse und Fertigkeiten in diesem neuen Umfeld notwendig sind. Die bekannte Forderung, ärztliches Know-how durch Management- und Ökonomiekompetenzen zu erweitern, ist grundsätzlich sicher richtig, doch stellt sich häufig für eine Ärztin und einen Arzt die Frage, welche spezifischen Inhalte aus diesen beiden grossen Fachbereichen für sie oder ihn relevant sind. Und nicht nur das, auch die Frage, welches die passenden und sinnvollen Wege und Weiterbildungen sind, um sich die ausgewählten Kompetenzen anzueignen. Natürlich existieren bereits heute zahllose Bildungsangebote renommierter Institutionen, doch viele Ärztinnen und Ärzte fordern zu Recht auch für die Bereiche Gesundheitsmanagement und -ökonomie Übersichtlichkeit, Systematik und klare Angaben zu Inhalten und Lernzielen.

Im Auftrag und in Zusammenarbeit mit der Verbindung der Schweizer Ärztinnen und Ärzte FMH bietet das College-M deshalb für Ärztinnen und Ärzte neue Weiter- und Fortbildungsangebote im Bereich Management und Qualitätsentwicklung an, welche mit den bestehenden Managementprogrammen des College-M inhaltlich und didaktisch abgestimmt sind (Abb.1):

\section{Managementgrundlagen für Ärztinnen und Ärzte}

Dieses Programm ist spezifisch für junge Ärztinnen und Ärzte in Spitälern (Assistenzärzte in der Weiterbildung), in Praxen und in anderen Gesundheitseinrichtungen konzipiert und orientiert sich eng an der beruflichen Funktion der teilnehmenden Ärztinnen und Ärzte.

Die wichtigsten Lernziele sind:

- Grundlegendes Wissen und Können in Führung und Management im eigenen Arbeitsumfeld. Vertrautheit mit der Bedeutung von Prozessen, Funktionen und Rollen.

- Entwickeln von Selbststeuerungsstrategien in wechselnden Arbeitskontexten und Erweitern der Fähigkeiten zu systematischer Reflexion von eigenen und fremden Erfahrungen, Ideen und Konzepten.

- Vertrautheit mit der Bedeutung unterschiedlicher Perspektiven, bzw. von fach- und berufsübergreifendem Denken und Handeln als Grundlage zu einem breiten Organisationsverständnis.

Das Programm gliedert sich in vier kurze Weiterbildungselemente à $1 \frac{1}{2}$ bzw. 2 Tage, deren wesentliche Inhalte sind:

- Element 1: Ärztliche Arbeitsprozesse, Funktionen und Perspektiven; Rollen des Arztes in Organisationen; Umgang mit Komplexität und Vielfalt.

- Element 2: Kommunikation und Kooperation; Zielgerichtete Steuerung von Prozessen.

Korrespondenz:

PD Dr. Peter Berchtold

College-M

Postfach

CH-3000 Bern 16
- Element 3: Entwicklungen im Gesundheitssystem; gesundheitsökonomische Grundlagen; Qualitätsmanagement und Patientenprozesse. 
- Element 4: Selbst- und Zeitmanagement; Persönliche Laufbahnplanungen.

Das Weiterbildungsprogramm «Managementgrundlagen" wird ab Herbst 2000 als Pilotprojekt durch die FMH finanziert und an drei Orten der Schweiz durchgeführt: je ein Programm für Ärztinnen und Ärzte des Universitätsspitals Zürich und des Inselspitals Bern sowie ein offenes Programm für alle weiteren Ärztinnen und Ärzte (siehe nachfolgende Übersicht).

\section{Die neuen Qualitätsmanagementprogramme}

Die neuen Basis- und Aufbauprogramme "Qualitätsmanagement» vermitteln die für Ärztinnen und Ärzte notwendigen Kenntnisse und Fähigkeiten zu Qualitätskonzepten und -instrumenten. Denn die Gewährleistung einer hohen Behandlungs- und Betreuungsqualität zählt immer mehr zu den wichtigsten Grundvoraussetzungen in der Gesundheitsversorgung. Und die zentrale Funktion der Ärztinnen und Ärzte in der Behandlung und Betreuung der Patienten sowie in der medizinischen Forschung und Entwicklung bedingt, dass beinahe jede Form der Qualitätsentwicklung auf ärztliche Initiativen oder mindestens ärztliches Mitwirken angewiesen ist.

Die Qualitätsmanagementprogramme gliedern sich in ein Basis- und ein Aufbauprogramm. Beide Stufen zeichnen sich durch einen hohen Bezug zu Interessen und Aufgaben der teilnehmenden Ärztinnen und Ärzte aus, indem neue Kenntnisse und Fähigkeiten anhand exemplarischer und eigener Projekte erworben werden.

Lernziele und Inhalte des Basisprogramms sind:

- Gesetzlicher Rahmen für Qualitätsmanagement;

- QE-Programm der FMH;

- Kenntnisse zu QM-Konzepten und -instrumenten: z.B. kontinuierliche QE, TQM, Q-Zirkel; Struktur-, Prozess- und Resultatqualität;

- Kenntnisse und Bewerten von Konzepten und Massnahmen zur Patientenprozessgestaltung und -steuerung;

- Kenntnisse über Evidence-based Practice: Bewertung und Kritik von Daten und Studien, Kennen bzw. Benutzen der Datenquellen;

- Kenntnisse der Grundlagen zu Kosteneffektivität,
Prozesskostenrechnungen;

- Kenntnisse zu Projektmanagementkonzepten;

- Verständnis des Zusammenspiels verschiedener Funktionen und Perspektiven in Organisationen als Grundlage jeder Qualitätsentwicklung.

Lernziele und Inhalte des Aufbauprogramms sind:

- Beurteilen und Aufbau von QM-Instrumenten;

- Kenntnisse zu Audits, Rollen externer Experten und Zertifizierungssysteme;

- Kenntnisse epidemiologischer Grundlagen/Modelle/Studiendesigns und statistischer Verfahren, Kenntnisse zu Evaluationsmodellen der Behandlungsqualität;

- Entwickeln und Bewerten von Massnahmen zur Patientenprozessgestaltung und -steuerung;

- Kenntnisse zu Prozesskostenrechnung, Kosteneffektivität und (pauschalen) Vergütungsformen;

- Kenntnisse zu Projektmanagementkonzepten und Organisationsmodellen, Einbetten von Projekten in Organisationen;

- Gestalten von Projektplanung, -ablauf, -steuerung;

- Entwickeln permanenter, zielgruppenadäquater Informationsprozesse;

- Basis- und Aufbauprogramme werden durch Fallstudien bzw. Erfahrungsbericht der Teilnehmerinnen und Teilnehmer ergänzt.

Das Basisprogramm umfasst $2 \frac{1}{2}$ Tage und richtet sich an jüngere Ärztinnen und Ärzte, die grundlegende QM-Kenntnisse und -Fähigkeiten suchen. Das Aufbauprogramm gliedert sich in 2 Elemente à je $2 \frac{1}{2}$ Tage und ist für Ärztinnen/Ärzte aus allen Bereichen und Institutionen geeignet, die sich erweiterte Kenntnisse und QM-Anwendungskompetenzen aneignen wollen.

\section{Zertifikate}

Die Managementgrundlagen- und Qualitätsmanagementprogramme schliessen mit einem Zertifikat der FMH ab. Voraussetzung für das FMH-QM-Zertifikat ist der Abschluss des ganzen QM-Curriculums (Basisund Aufbauprogramm).

Die Schaffung eines Fähigkeitsausweis im Rahmen der FMH-Weiterbildungsordnung ist in Vorbereitung. 


\begin{tabular}{|c|c|}
\hline \multicolumn{2}{|c|}{ Managementgrundlagen für Ärztinnen und Ärzte } \\
\hline \multicolumn{2}{|l|}{ Daten } \\
\hline $\begin{array}{l}\text { Element } 1 \\
1,5 \text { Tage }\end{array}$ & 15./16. September 2000 \\
\hline $\begin{array}{l}\text { Element } 2 \\
2 \text { Tage }\end{array}$ & 30. November / 1. Dezember 2000 \\
\hline $\begin{array}{l}\text { Element } 3 \\
1,5 \text { Tage }\end{array}$ & 26./27. Januar 2001 \\
\hline $\begin{array}{l}\text { Element } 4 \\
1,5 \text { Tage }\end{array}$ & 23./24. März 2001 \\
\hline \multicolumn{2}{|c|}{$\begin{array}{l}\text { Christof Schmitz, Corporate Pragmatics, Zürich } \\
\text { Susanne Quistorp, Zürich }\end{array}$} \\
\hline \multicolumn{2}{|c|}{$\begin{array}{l}\text { 1) Offenes Programm: Stadt Zürich } \\
\text { 2) Programm für das Universitätsspital Zürich: } \\
\text { Stadt Zürich } \\
\text { 3) Programm für das Inselspital Bern: College-M, } \\
\text { Bern }\end{array}$} \\
\hline \multicolumn{2}{|c|}{ Qualitätsmanagement } \\
\hline \multicolumn{2}{|l|}{ Daten } \\
\hline $\begin{array}{l}\text { 1. Durchführung } \\
2,5 \text { Tage }\end{array}$ & 17.-19. August 2000 \\
\hline $\begin{array}{l}\text { 2. Durchführung } \\
2,5 \text { Tage } \\
\text { Aufbauprogramm }\end{array}$ & 19.-21. Oktober 2000 \\
\hline $\begin{array}{l}\text { Element } 1 \\
3 \text { Tage }\end{array}$ & 23.-25. November 2000 \\
\hline $\begin{array}{l}\text { Element } 2 \\
3 \text { Tage }\end{array}$ & 11.-13. Januar 2001 \\
\hline $\begin{array}{l}\text { Verantwortliche I } \\
\text { Peter Berchtold, C } \\
\text { Hans Heinrich Bru } \\
\text { Richard Cranovsk }\end{array}$ & $\begin{array}{l}\text { Programmleiter } \\
\text { College-M, Bern } \\
\text { unner, FMH, Bern } \\
\text { ky, FMH, Bern }\end{array}$ \\
\hline $\begin{array}{l}\text { Ort } \\
\text { Stadt Bern }\end{array}$ & \\
\hline $\begin{array}{l}\text { Kosten } \\
\text { Basisprogramm } \\
\text { Aufbauprogramm }\end{array}$ & $\begin{array}{l}\text { Fr. 1050.- } \\
\text { Fr. 1850.- }\end{array}$ \\
\hline
\end{tabular}

\section{Managementprogramm FE1}

Daten

Element 1 21.-23. August 2000

3 Tage

Element 2 11.-13. September 2000

3 Tage

Element 3 18.-21. Oktober 2000

4 Tage

Verantwortliche Programmleiter

Suzanne Lüthi, College-M, Bern

Erna Walt, Basel

Ort

College-M, Bern

Kosten

Fr. 3500.-

\section{Managementprogramm FE2}

\section{Daten}

Element $1 \quad$ 16.-18. Oktober 2000

3 Tage

Element 2 8.-10. November 2000

3 Tage

Element 3 13.-16. Dezember 2000

4 Tage

Element 4 18.-20. Januar 2001

3 Tage

Element 5 28. Februar-2. März 2001

3 Tage

Element 6 5.-6. April 2001

2 Tage

Verantwortliche Programmleiter

Peter Berchtold, College-M, Bern

Christof Schmitz, Corporate Pragmatics, Zürich

Ort

College-M, Bern

Kosten

Fr. 7920.-

\section{Name}

Vorname

Institution

Adresse

PLZ / Ort

Telefon

E-mail 


\section{Facharztprüfung zur Erlangung des Facharzttitels FMH für Kardiologie}

Aufgrund des Weiterbildungsprogrammes und des Beschlusses des Zentralvorstandes der FMH vom 3. Februar 1999 ist das Bestehen der Facharztprüfung für alle Kandidatinnen und Kandidaten, welche ihre Weiterbildung nach dem 31. Dezember 2000 abschliessen, Voraussetzung zur Erlangung des Facharzttitels FMH für Kardiologie. Näheres zu den Übergangsbestimmungen und Ausnahmen ist in der Schweizerischen Ärztezeitung Nr. 5 vom 2. Februar 2000 publiziert.

Ort: Kardiologische Abteilung, Kantonsspital, Basel Datum/Zeit: Donnerstag, 7. Dezember 2000, 8.00 Uhr

Prüfungsgebühr: Die SGK erhebt eine Prüfungsgebühr von Fr. 500.-.

Anmeldung (unter Beilage des Curriculum vitae): Prof. Alberto Righetti, Centre et Division de Cardiologie, Hôpital Cantonal, 1211 Genève 14, Tel. 02237272 02, Fax 0223727229

Anmeldefrist: 30. September 2000

Ort: CHUV, Lausanne

Datum: Freitag, 25. August 2000

Prüfungsgebühr: Die SGN erhebt eine Prüfungsgebühr von Fr. 700.- für Mitglieder, Fr. 1200.- für Nichtmitglieder.

Anmeldung: Prof. Dr. med. Jan Müller-Brand, Universitätsklinik und Institut für Nuklearmedizin, Kantonsspital, 4031 Basel, Tel. 06126547 05, Fax 06126549 25, E-mail: jmueller@uhbs.ch

Anmeldefrist: 15. Juli 2000

dispositions transitoires et les dérogations, veuillez vous référer à la publication du Bulletin des médecins suisses no 5, du 2 février 2000.

Lieu: Centre de Division de Cardiologie, HUG, Genève

Date: Jeudi 7 décembre 2000, à 8 h

Inscriptions (avec soumission du curriculum vitae): Prof. Alberto Righetti, Centre et Division de Cardiologie, Hôpital Cantonal, 1211 Genève 14, tél. 02237272 02, fax 0223727229

Délai d'inscription: 30 septembre 2000

Lieu: CHUV, Lausanne

Date: Vendredi, le 25 août 2000

Taxe d'examen: La SSMN prélève une participation aux frais de Fr. 700.- pour les membres, Fr. 1200.pour les non-membres.

Inscription: Prof. Dr Jan Müller-Brand, Universitätsklinik und Institut für Nuklearmedizin, Kantonsspital, 4031 Basel, tél. 06126547 05, fax 061 26549 25, e-mail: jmueller@uhbs.ch

Délai d'inscription: le 15 juillet 2000
Conditions d'admission: Réussite de la première partie de l'examen. Il est recommandé de passer l'examen de spécialiste au plus tôt durant la dernière année de la formation postgraduée réglementaire (art. 22 RFP). 